\title{
Brain Tissue Classification from Multispectral MRI by Wavelet based Principal Component Analysis
}

\author{
Sindhumol S, Kannan Balakrishnan \\ Department of Computer Applications, Cochin University of Science and Technology \\ Kochi, Kerala, India \\ sindhumol09@gmail.com \\ Anil Kumar \\ Institute of Radiology and Imaging Sciences, Indira Gandhi Co-operative Hospital \\ Kochi, Kerala, India \\ anil.k@medall.in
}

\begin{abstract}
In this paper, we propose a multispectral analysis system using wavelet based Principal Component Analysis (PCA), to improve the brain tissue classification from MRI images. Global transforms like PCA often neglects significant small abnormality details, while dealing with a massive amount of multispectral data. In order to resolve this issue, input dataset is expanded by detail coefficients from multisignal wavelet analysis. Then, PCA is applied on the new dataset to perform feature analysis. Finally, an unsupervised classification with Fuzzy C-Means clustering algorithm is used to measure the improvement in reproducibility and accuracy of the results. A detailed comparative analysis of classified tissues with those from conventional PCA is also carried out. Proposed method yielded good improvement in classification of small abnormalities with high sensitivity/accuracy values, 98.9/98.3, for clinical analysis. Experimental results from synthetic and clinical data recommend the new method as a promising approach in brain tissue analysis.
\end{abstract}

Index Terms - Fuzzy C-Means Clustering, Magnetic Resonance Imaging, Multisignal wavelet analysis, Multispectral analysis, Principal Component Analysis

\section{INTRODUCTION}

Magnetic Resonance Imaging (MRI) is a multi sequence digital imaging technique in which stacks of images are acquired with different tissue contrasts. For example, T1-weighted sequence gives considerable contrast between Gray Matter (GM) and White Matter (WM). T2-weighted sequence shows details of Cerebrospinal Fluid (CSF) and abnormalities, whereas FLAIR images suppress CSF effects to give hyperintense lesions details. Simultaneous analysis of these sequences to collect the prominent pathological information is a great challenge in clinical analysis [1, 2]. Methods using multispectral approaches are useful in this context to improve the accuracy and consistency of the clinical results. A typical multispectral analysis system is depicted in Figure 1. Image ${ }_{1}$ Image $_{2} \ldots$ Image $_{n}$ are images acquired in band $_{1}$, band ${ }_{2} \ldots$ band $_{n}$ respectively. Required input bands are collected from available data, after preprocessing steps like denoising, registration and contrast enhancement. Classification results are highly dependent on the accuracy of extracted features. So, feature extraction and selection [2] has an important role in multispectral analysis. In Figure $1, \mathrm{f}_{1}, \mathrm{f}_{2} \ldots \mathrm{f}_{\mathrm{k}}$, represents the best features selected for training and classification to give final outputs, Class ${ }_{1}$, Class $_{2} \ldots$ Class $_{m}$.

Transform based methods like Principal Component Analysis (PCA) [3], Independent Component Analysis (ICA) [4], Nonnegative Matrix Factorization (NMF) [5] and their extensions [6-8] are widely used in redundancy reduction and feature extraction from multispectral images. In recent years, brain MRI studies using PCA [911] have shown great advance in normal and abnormal tissue analysis. One major deficiency of PCA is its global orthogonal representation, which limits the extraction of local characteristics [8]. In other words, PCA is good only at extracting the most frequently occurred features, which makes it difficult to extract small details like presence of tumours/lesions from background dominating components [8, 12]. Researchers have proposed several methods in recent past to resolve this issue. Spectral angle based clustering approaches before dimensionality reduction [12, 13] showed good performance in local feature analysis. But, results were found to be varying on spectral angle settings. Wavelet based attempts were made in $[14,15]$ to retain local information by wavelet denoising of each pixel's spectra, followed by downsampling to reduce the dimensionality of spectra. However, information loss due to reconstruction from selected subbands is not acceptable in MRI clinical trials, where maximum accuracy is always expected for pathology analysis. 
A new method, multisignal wavelet based Principal Component Analysis, is proposed in this work for

improved brain tissue analysis from multispectral MRI. To

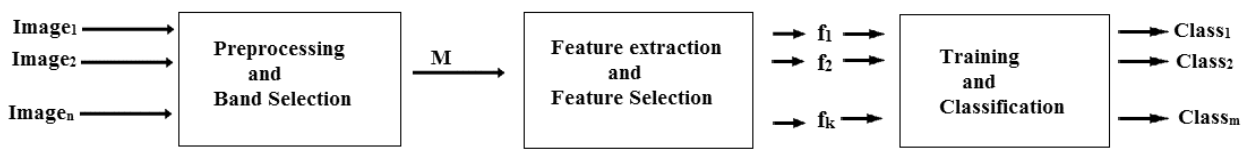

Figure 1. A typical multispectral classification system

avoid the loss of less frequently occurred objects like small lesions while dealing with massive amount of information, a multisignal wavelet analysis is applied on input data prior to applying PCA. Mutually independent high frequency components (detail coefficients) [14] from wavelet analysis are selected to expand the input data set. Best Principal Components (PCs) are selected based on maximum explained variance [8] provided by each PC. Unsupervised classification method, Fuzzy C-Means clustering (FCM) [16, 17], is finally applied on the selected PCs for segmentation and classification. Visual and quantitative analysis using synthetic and clinical data was conducted to evaluate the improvement in tissue classification by proposed method over conventional PCA. Experimental results supported the proposed method as a potential approach in simultaneous brain tissue analysis from different MRI sequences.

The rest of the paper is organized as follows. The materials, mathematical concepts and proposed method are described in section II. Experimental results and analysis are summarized in section III. Section IV concludes the paper.

\section{MATERIALS AND METHODS}

\section{A. Input dataset}

Both synthetic and clinical images were considered in the evaluation of the proposed system. Synthetic MR images were collected from BrainWeb [18] database. 12 slices (slice no.98-109) of abnormal data set, containing Multiple Sclerosis (MS) details were selected to form the multispectral suite. Axial T1-Weighted Images (T1WI), T2-Weighted Images (T2WI), and Proton Density Images (PDI) with parameter settings 1-mm slice thickness, intensity non-uniformity $20 \%$ and noise level $0 \%$ were included in the set.

Axial T1WI, T2WI and FLAIR images collected from 20 abnormal cases were considered for clinical image analysis. These images were sampled by Siemens' whole body 3 Tesla (T) MR system (Siemens, AG Medical Solutions, Erlangen, Germany). Three sample slices from each MRI sequence showing traces of abnormal tissues were selected for each case; Total 20x3 multispectral image cubes were included in the analysis. The clinical cases were acquired by axial spin echo T1WI with repetition time $(\mathrm{TR})=1600 \mathrm{~ms}$, echo time $(\mathrm{TE})=8.9 \mathrm{~ms}$ and T2WI with TR/TE $=4000 \mathrm{~ms} / 95 \mathrm{~ms}$. FLAIR images have $\mathrm{TR} / \mathrm{TE}=6000 \mathrm{~ms} / 94 \mathrm{~ms}$, inversion time $(\mathrm{TI})=$ 2026.5ms. Additional parameters includes, slice gap, $6.5 \mathrm{~mm}$, thickness, $5 \mathrm{~mm}$, and the matrix size $227 \times 260$ pixels.

\section{B. Multisignal Wavelet Analysis}

Images in input multispectral cube are reshaped into a set of 1-d signals, and a collection of those signals forms MRI multisignal [14] stored as a matrix organized row wise (or column wise). Wavelet transform of a signal can be calculated by projection of the signal onto shifted and scaled version of a basic function. In the case of higher dimensional signals, it can be done in spatial or spectral direction [15]. Mallat's multiresolution algorithm [19] for 1-d signal analysis is shown in Figure 2 [20]. An extension of Mallat's algorithm in spectral dimension [14] is used in this work for wavelet analysis.

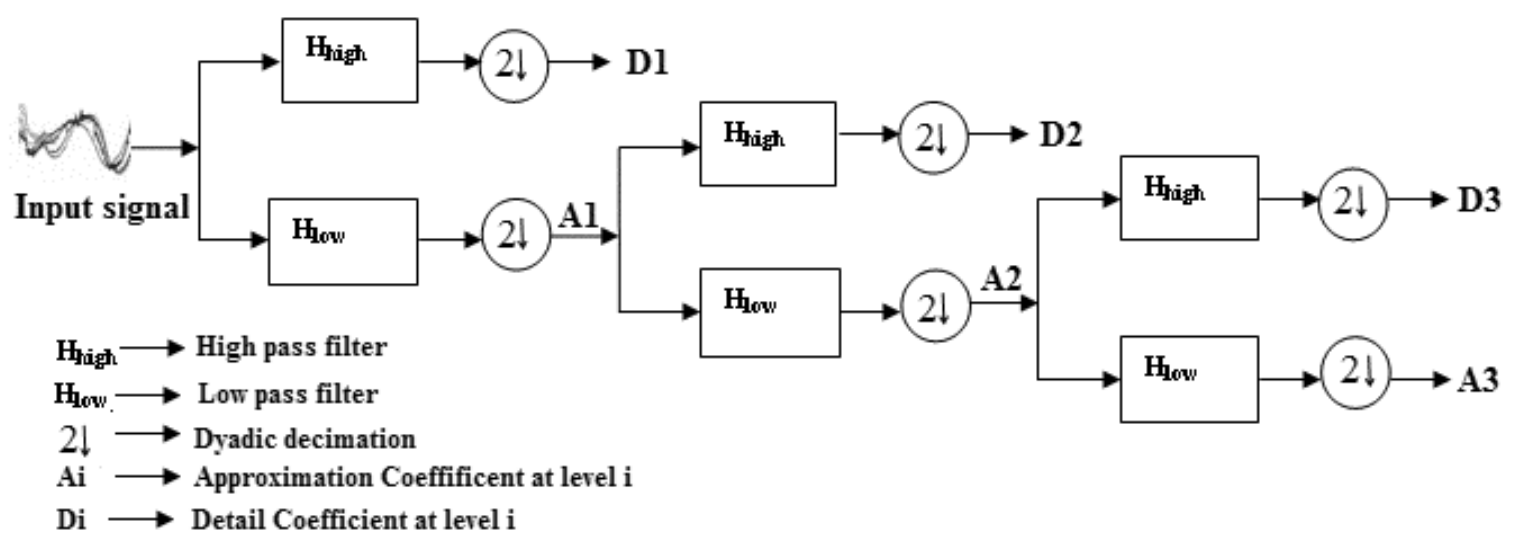

Figure 2. Multiresolution analysis of input multisignals [20]

In wavelet analysis [19] of spectra, a low pass filter and its corresponding high pass filter are simultaneously applied on input multisignal at each level 'i' in its spectral domain[14, 20]. Spectra are decomposed into approximation coefficient $A_{i}$ and detail coefficient $D_{i}$ as shown in Figure 2. A dyadic decimation is applied on both 
coefficients, which reduces the original resolution into half of its original length and eliminates the irrelevant elements involved in the signals. This procedure is recursively applied on approximation coefficients, $\mathrm{A}_{\mathrm{i}}$, to give increasingly smoother versions of original signals [20]. Both low frequency and high frequency features can be preserved through the explained multiresolution analysis. Studies show that high frequency subband signals are independent and low frequency subbands are weakly dependent [14]. In this work, only the local characteristics are required to preserve with high priority. Decorrelated high frequency subbands coefficients are used in the input multisignal band expansion to achieve this.

\section{Principal Component Analysis}

Principal Component Analysis (PCA) is perhaps the most popular dimensionality reduction technique [3]. The main use of PCA is to reduce the dimensionality of a dataset, while retaining as much information as possible in resulting PCs in descending order of information content. It is often the case that only a small number of these components contain the effective information needed. It is a multivariate procedure, in which a rotation is found so that the first axis corresponding to the first component is rotated to the direction where the variance of the data set is greatest $[3,9,10]$. The next component will then be the direction perpendicular to the first with the most variance and so on. Essentially, a set of correlated variables is transformed into a set of uncorrelated variables, which are ordered by reducing variability. The uncorrelated PCs are linear combinations of the original variables, and the last of these PCs can be avoided in feature selection.

To do PCA computation, input vectors are first normalized to have zero mean and unity variance. A step by step description of PCA algorithm is best explained in [3, 21]. A detailed explanation of PCA computation from brain MRI images is available from [21].

\section{Fuzzy C-Means Clustering}

Fuzzy C-Means (FCM) clustering is an unsupervised classification technique introduced by Bezdek in 1981 [17], where each data point belongs to a cluster with some degree that is specified by a fuzzy membership grade [22]. Let $\mathbf{X}=\left(\mathrm{x}_{1}, \mathrm{x}_{2}, . ., \mathrm{x}_{\mathrm{N}}\right)$ denotes an input image with ' $N$ ' pixels to be partitioned into ' $c$ ' clusters. $x_{i}$ represents multispectral (features) data, and 'c' is the number of clusters with $2 \leq \mathrm{c}<\mathrm{N}$. The standard FCM function for partitioning a dataset $\left\{x_{k}\right\}_{k=1}^{N}$ into 'c' clusters is given by [23],

$$
\mathbf{J}_{\mathrm{m}}=\sum_{i=1}^{c} \sum_{k=1}^{N} U_{i k}^{m}\left\|x_{k}-v_{i}\right\|^{2}
$$

In (1), $\left\{v_{i}\right\}_{i=1}^{c}$ are the centres of clusters, and the array, $\left\{U_{i k}\right\}, i=1 \ldots c, k=1, \ldots N$, is a partition matrix such that

$$
U \in\left\{U_{i k} \in[0,1] \mid \sum_{i=1}^{c} U_{i k}=1, \forall k\right.
$$

$$
\left.\& \quad 0<\sum_{k=1}^{N} U_{i k}<\mathrm{N}, \forall i\right\}
$$

The parameter ' $m$ ' is a weighting exponent on each fuzzy membership. It determines fuzziness amount of the resulting classification. Gray level values are the commonly used features in image processing. When high membership values are assigned to pixels, whose intensities are close to the centroid of its particular class, FCM objective function is minimized. Low membership values are assigned when the point is far from the centroid [23]. Algorithm starts with an initial guess for each cluster centre, and it converges to a solution for ' $v_{i}$ ' representing the local minimum of the objective function. A detailed explanation of FCM theorem and algorithm is available in [23].

\section{E. Proposed Method}

Wavelet decomposition of the multispectral data and PCA are the main theoretical concepts used in this algorithm. Figure 3 explains the main steps involved in it. Co-registered images from multiple MRI sequences form the input multispectral suite. Each pixel is considered as a vector representing the spectral signature of the area specified by that pixel. Input multispectral image is then reshaped into a collection of 1-d spectral signatures as shown in Figure 3. 1-d multisignal wavelet decomposition is applied on these signals, which divides the spectral domain into low frequency and high frequency components as described in section II B. Number of bands in input multisignal is then expanded by appending detail coefficients from subband components. Standard normalization is performed on the expanded dataset, and PCA is applied on the normalized signals. Components yielding cumulative explained variance greater than a specified threshold, $\alpha$, are selected as the candidates for classification, and FCM clustering algorithm is followed to classify the brain matters automatically. The proposed algorithm can be summarized as follows:

1. Input: Co-registered images $\mathrm{I}_{1}, \mathrm{I}_{2}, \mathrm{I}_{3}, \mathrm{I}_{4} \ldots$ from MRI sequences T1WI, T2WI, PDI, FLAIR etc.

2. Multispectral image formation, $\mathbf{M}=\left[\mathrm{I}_{1}, \mathrm{I}_{2}, \mathrm{I}_{3}\right.$, $\left.\mathrm{I}_{4} \ldots \mathrm{I}_{\mathrm{p}}\right]$, where ' $\mathrm{p}$ ' denotes number of input images.

3. Reshape each image to 1-d signal to form input multisignal $\mathbf{X}$.

$$
\begin{aligned}
\mathbf{X}=\left[\operatorname{reshape}\left(\mathrm{I}_{1}\right) ; \operatorname{reshape}\left(\mathrm{I}_{2}\right) ;\right. & \left.\operatorname{reshape}\left(\mathrm{I}_{3}\right) ; \ldots \operatorname{reshape}\left(\mathrm{I}_{\mathrm{p}}\right)\right]
\end{aligned}
$$

4. Apply 1-d multisignal wavelet analysis on $\mathbf{X}$ to decompose the signals into approximation coefficients, $\mathbf{A}$, and detail coefficients, $\mathbf{D}$.

5. Expand input data $\mathbf{X}$ by appending $\mathbf{D}$ and normalize it. Modified input multisignal, $\mathbf{X}_{\text {new }}=$ Normalize $([\mathbf{X} ; \mathbf{D}])$

6. Apply PCA on $\mathbf{X}_{\text {new }}$,

$\left[\mathbf{P C}_{1}, \mathbf{P C}_{2}, \mathbf{P C}_{3}, \ldots, \mathbf{V}\right]=\operatorname{PCA}\left(\mathbf{X}_{\text {new }}\right)$.

$\mathbf{P C}_{1}, \mathbf{P C}, \mathbf{P C}_{3}$ are generated $\mathrm{PCs}$ in the order of decreasing explained variance. ' $\mathbf{V}$ ' 
represents the explained variance vector corresponding to the output PCs.

7. Select ' $\mathrm{k}$ ' minimum number of PCs such that

$$
\sum_{i=1}^{k} V_{i} \geq \alpha \text {, where } V_{i} \text { represents explained }
$$
variance by $i^{\text {th }} \mathrm{PC}$.

8. Reshape each selected PC to corresponding 2-d form, and apply FCM.

9. Label the clusters from step 8 into different brain tissues.

\section{F. Performance Measures}

Tanimoto Index, sensitivity, specificity, accuracy and False Positive Rate (FPR) are the performance measures used in quantitative analysis in this study. Tanimoto Index (TI) is the most commonly used measurement in medical imaging [20], and it can be measured by comparing the reproduced tissues with ground truth using the formula,

$$
T=\frac{|A \cap B|}{|A \cup B|}
$$

In (2), ' $A$ ' and ' $B$ ' are two datasets involved in the classification comparison. In this work, similarity checking of reproduced brain tissues with corresponding ground truth is performed using (2).

Another set of statistical measurements based on error rate calculations are also considered. Sensitivity, Specificity, Accuracy, and False Positive Rate (FPR) are calculated from confusion matrix [20,24] as follows:
Sensitivity $=(\mathrm{TP} /(\mathrm{TP}+\mathrm{FN})) * 100 \%$

Specificity $=(\mathrm{TN} /(\mathrm{TN}+\mathrm{FP})) * 100 \%$

Accuracy $=((\mathrm{TP}+\mathrm{TN}) /(\mathrm{TP}+\mathrm{TN}+\mathrm{FP}+\mathrm{FN})) * 100 \%$

$\mathrm{FPR}=\mathrm{FP} /(\mathrm{TN}+\mathrm{FP}) * 100 \%$, where True Positive (TP) - Tumor pixels correctly identified as tumor, False positive (FP) - Other tissues incorrectly identified as tumor, True Negative (TN) - Other tissues correctly identified and False Negatives (FN) - Tumor pixels incorrectly identified as others.

\section{RESULTS AND DISCUSSION}

The system for evaluation of proposed method was implemented in Matlab 7. Both synthetic and clinical MRI images, described in section II A, were used to conduct the experiments. Matlab based manual image registration was applied on clinical data to generate coregistered multispectral images. Functions from Matlab Wavelet toolbox helped to do multisignal analysis of 1-d input signals. Daubechies 2 ('db2') wavelets were selected by trial and error for multisignal analysis, since it was found to be yielding good results for input multispectral data. Functions in Statistics Toolbox were used in PCA, and automatic segmentation of the selected components was done with FCM method in Fuzzy Logic Toolbox. In this work, cumulative explained variance threshold, $\alpha$, is set as $98 \%$. Experiments for proposed method and conventional PCA were performed in the same environment, and results are presented in the following sections.

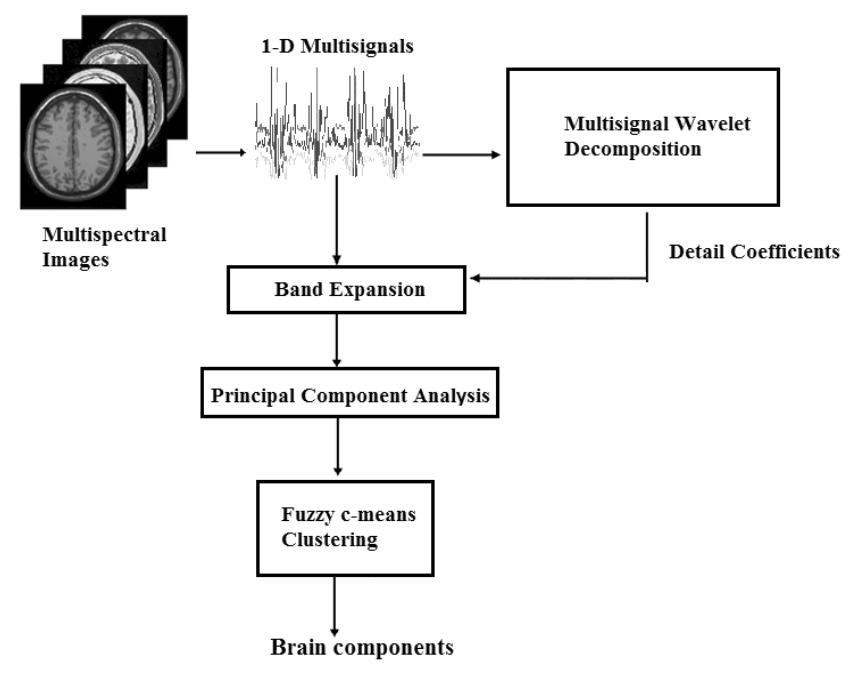

Figure 3. Proposed method

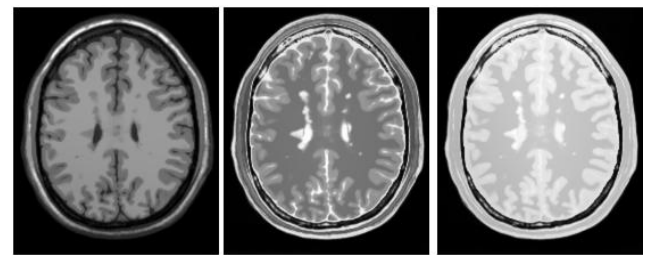

(a). Synthetic Images T1WI, T2WI and PDI (from left to right)

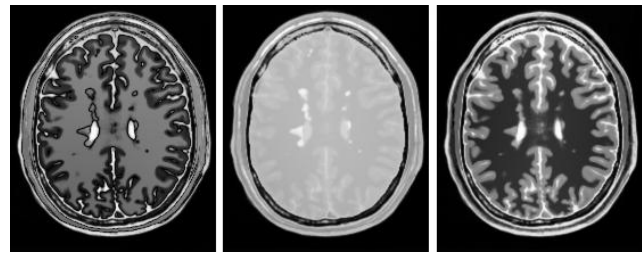

(b). Principal Components from PCA 


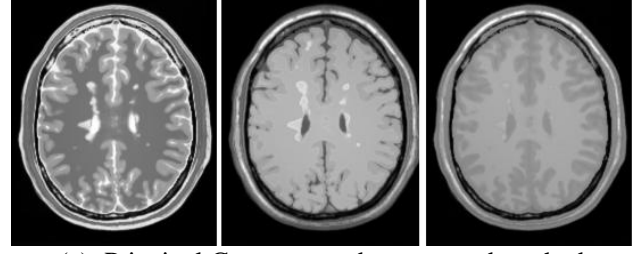

(c). Principal Components by proposed method
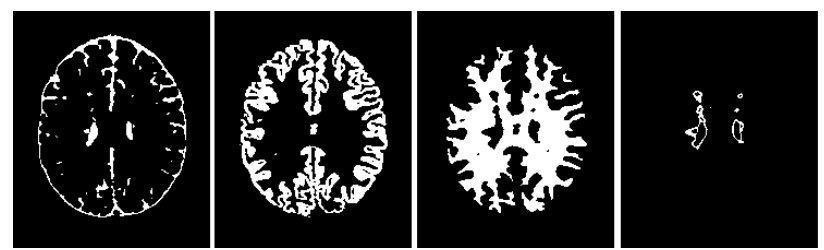

(d). Classified brain tissues from conventional PCs
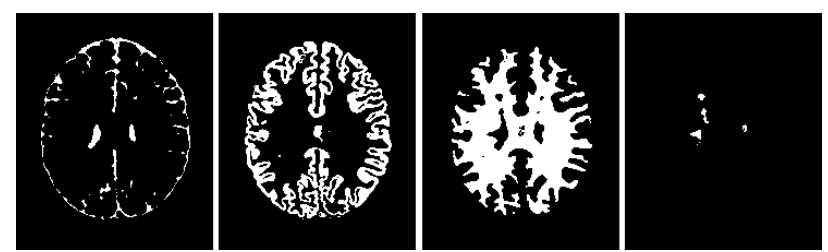

(e). Classified brain tissues by proposed method

Figure 4. Principal components and classified results

\section{A. Synthetic Image Analysis}

Twelve sample slices from axial T1WI, T2WI, and PDI with parameter settings as described in section II A were considered in the analysis. For example, input images from slice no. 102 from Brainweb data is shown in Figure 4(a). WM and GM components are clear from T1WI. T2WI shows CSF and White Matter Lesion (WML) details, and PD image gives additional information on abnormal features. PCs from PCA and proposed method are given in Figure 4(b) and Figure 4(c) respectively. From dimensionality reduction process, it is

TABLE I. QUANTITATIVE ANALYSIS OF SYNTHETIC IMAGES

\begin{tabular}{|c|c|c|c|c|c|}
\hline \multirow{2}{*}{ Tissue } & Analysis & $\begin{array}{c}\text { Sensiti } \\
\text { vity }\end{array}$ & $\begin{array}{c}\text { Specifi } \\
\text { city }\end{array}$ & $\begin{array}{c}\text { Accura } \\
\text { cy }\end{array}$ & TI \\
\hline \multirow{2}{*}{$C S F$} & PCA+FCM & 98.1 & 98.0 & 98.0 & 0.69 \\
\cline { 2 - 6 } & $\begin{array}{c}\text { Proposed } \\
\text { Method }\end{array}$ & 95.4 & 99.4 & 98.7 & 0.71 \\
\hline \multirow{2}{*}{$W M$} & PCA+FCM & 99.7 & 96.1 & 96.7 & 0.86 \\
\cline { 2 - 6 } & $\begin{array}{c}\text { Proposed } \\
\text { Method }\end{array}$ & 99.8 & 98.5 & 98.4 & 0.93 \\
\hline \multirow{2}{*}{$G M$} & PCA+FCM & 89.7 & 97.4 & 96.2 & 0.77 \\
\cline { 2 - 6 } & $\begin{array}{c}\text { Proposed } \\
\text { Method }\end{array}$ & 98.8 & 97.1 & 97.3 & 0.83 \\
\hline \multirow{2}{*}{$W M L$} & PCA+FCM & 64.9 & 32.1 & 47.8 & 0.37 \\
\cline { 2 - 6 } & $\begin{array}{c}\text { Proposed } \\
\text { Method }\end{array}$ & 64.3 & 99.7 & 96.3 & 0.48 \\
\hline
\end{tabular}

found that majority of the information is accumulated in first two PCs. First three PCs in the order of decreasing explained variance were selected for classification process. From Figure 4(c) it is seen that details of the brain features extracted by proposed method are more specific compared to results from conventional PCA, especially in the case small abnormalities like WML. To visually analyze the effect of the new method in classification, results from FCM clustering are summarized in Figure 4(d) and 4(e), in the order of CSF, GM, WM and WML from left to right. Reproduced tissues from PCA, shown in Figure 4(d), fails to keep the accuracy of the classified brain tissues. On comparing each column, the improvement in accuracy by wavelet based PCA is clearly visible without any indexing. WML comparison, as shown in the last column, emphasizes the positive impact of the method in small object classification from massive amount of data.

Improvement in classification by proposed method was further evaluated by a detailed quantitative analysis on 12 synthetic multispectral sets, and results are summarized in Table I. Average sensitivity, specificity, accuracy, and Tanimoto Index (TI) values were measured for CSF, GM, WM and WML. The same experiment was repeated for extracted features from conventional PCA also, and observed results are included in Table I. Quantitative measures confirmed the proposed method as an efficient approach for classification. A drastic increase in Tanimoto index and accuracy is found in the case of WML, accuracy increased from 47.8 to 96.3 and Tanimoto index improved from 0.37 to 0.48 . Synthetic image analysis supports proposed method, with good visual and quantitative results for normal and abnormal tissues. To evaluate the potential of the new method in real image analysis, abnormal data taken from 20 clinical cases were considered, and experimental results are summarized in the next section.

\section{B. Clinical Image Analysis}

Axial T1WI, T2WI and FLAIR images, with parameters and specification as discussed in section II A, were selected for real image analysis. Three abnormal slices were considered from each MRI sequence for each case, and total 20x3 multispectral sets were formed for

TABLE II.
\begin{tabular}{|c|c|c|c|c|}
\hline \multirow{2}{*}{ Cases } & $\begin{array}{c}\text { Feature } \\
\text { Analysis }\end{array}$ & PC1 & PC2 & PC 3 \\
\hline \multirow{2}{*}{1} & PCA & 58.5 & 34.4 & 7.1 \\
\cline { 2 - 5 } & $\begin{array}{c}\text { Proposed } \\
\text { Method }\end{array}$ & 73.9 & 20.7 & 3.5 \\
\hline \multirow{2}{*}{2} & PCA & 55.1 & 37.1 & 7.9 \\
\cline { 2 - 5 } & $\begin{array}{c}\text { Proposed } \\
\text { Method }\end{array}$ & 70.3 & 23.6 & 4.2 \\
\hline \multirow{2}{*}{3} & PCA & 64.5 & 23.0 & 12.5 \\
\cline { 2 - 5 } & $\begin{array}{c}\text { Proposed } \\
\text { Method }\end{array}$ & 76.9 & 12.8 & 8.4 \\
\hline \multirow{2}{*}{4} & PCA & 48.9 & 26.6 & 24.5 \\
\cline { 2 - 5 } & $\begin{array}{c}\text { Proposed } \\
\text { Method }\end{array}$ & 62.2 & 20.6 & 15.4 \\
\hline \multirow{2}{*}{5} & PCA & 44.5 & 36.3 & 19.3 \\
\cline { 2 - 5 } & $\begin{array}{c}\text { Proposed } \\
\text { Method }\end{array}$ & 59.8 & 27.2 & 11.3 \\
\hline
\end{tabular}




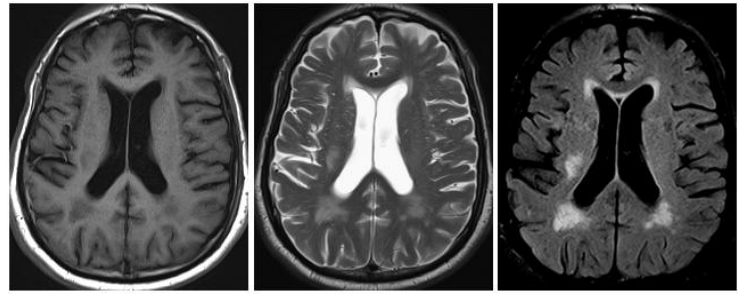

(a). Input clinical Images T1WI, T2WI and FLAIR (left to right)
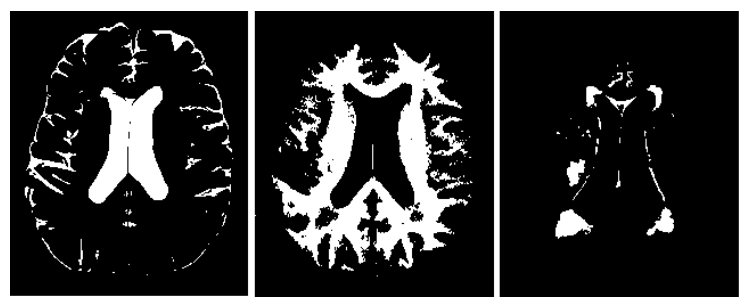

(b). Classified brain tissues from conventional PCs
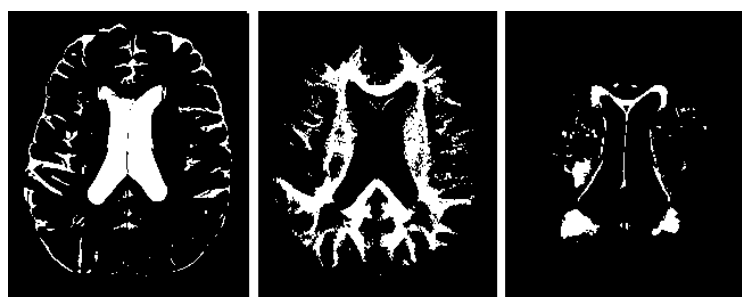

(c). Classified brain tissues from wavelet based PCs

Figure 5. Classified results from clinical images

analysis. Proposed method is applied on co-registered images from these sets, and FCM classification was performed on selected PCs. These experiments were repeated for conventional PCA based classification, and explained variances for first three components are summarized in Table II. Proposed method accumulated most of the variances in first two components, whereas not much difference observed between first two components from PCA. The wavelet based PCA

\begin{tabular}{|c|c|c|c|c|c|}
\hline TABLE III. & \multicolumn{1}{c}{ QUANTITATIVE ANALYSIS OF CLINICAL IMAGES } \\
\hline \multirow{2}{*}{ Tissue } & Analysis & $\begin{array}{c}\text { Sensit } \\
\text { ivity }\end{array}$ & $\begin{array}{c}\text { Specif } \\
\text { icity }\end{array}$ & $\begin{array}{c}\text { Accu } \\
\text { racy }\end{array}$ & FPR \\
\hline \multirow{2}{*}{$\boldsymbol{C S F}$} & PCA+FCM & 85.6 & 99.7 & 97.2 & 0.3 \\
\cline { 2 - 6 } & $\begin{array}{c}\text { Proposed } \\
\text { Method }\end{array}$ & 98.6 & 99.8 & 99.6 & 0.2 \\
\hline \multirow{3}{*}{ Lesions } & PCA+FCM & 92.1 & 91.1 & 91.8 & 8.9 \\
\cline { 2 - 6 } & $\begin{array}{c}\text { Proposed } \\
\text { Method }\end{array}$ & 94.1 & 97.6 & 96.3 & 2.4 \\
\cline { 2 - 6 } & $\begin{array}{c}\text { PCA+FCM } \\
\text { Method }\end{array}$ & 64.5 & 98.1 & 97.3 & 1.9 \\
\hline
\end{tabular}

generates more than three PCs depending on the number of bands in the expanded data set. However, more than $98 \%$ of the variance was found to be distributed in first three components, and they were selected for classification in this analysis. To analyze the visual results in clinical case, Figure 5(a) shows sample slices from T1WI, T2WI and FLAIR sequences. T1WI shows WM details; T2WI gives CSF and abnormal points, and
FLAIR images specify abnormal tissues. GM information is found to be very difficult to extract from these images. Results from PCA based FCM is summarized in Figure 5(b), and those from wavelet based PCA and FCM using 'db2' wavelet are shown in Figure 5(c). Reproduced results are presented in the order of CSF, WM and abnormality from left to right. Improvement in tissue classification by proposed method can be best explained by column wise comparison of Figure 5(b) and 5(c). It is very clear from CSF comparison that more information is available from proposed method. Visual results supported the new method in local object analysis, with comparatively more accurate WM and abnormal tissues compared to those from PCA. Figure 5(c) shows that abnormalities observed from FLAIR image and proposed method are very similar, and its presence in WM is well explained visually by proposed method. A detailed performance analysis using measurements like sensitivity, specificity, accuracy and TI values was also conducted to ensure the effect of the proposed method in MRI analysis. Average values of measurements from 60 multispectral slice sets are summarized in Table III.

Results in Table III confirm the efficiency of the new method in brain matter analysis, with positive differences for all tissues. In the case of small lesions, average sensitivity value improved from 64.5 to 98.9 . FPR values were observed to be less for proposed method. Considerable reduction in false positive rate explains the robustness and reliability of the new approach in clinical trials. To study the variation of TI values for reproduced tissues, average results from three multispectral slice sets were considered for each case. Measured values for 10 cases are plotted in Figure 6. Top row shows the results for CSF, middle row gives TI measurements for WM and last row describes the results for abnormal tissues. Improvement in TI values implies the increased similarity of the results to the groundtruth. In the case of

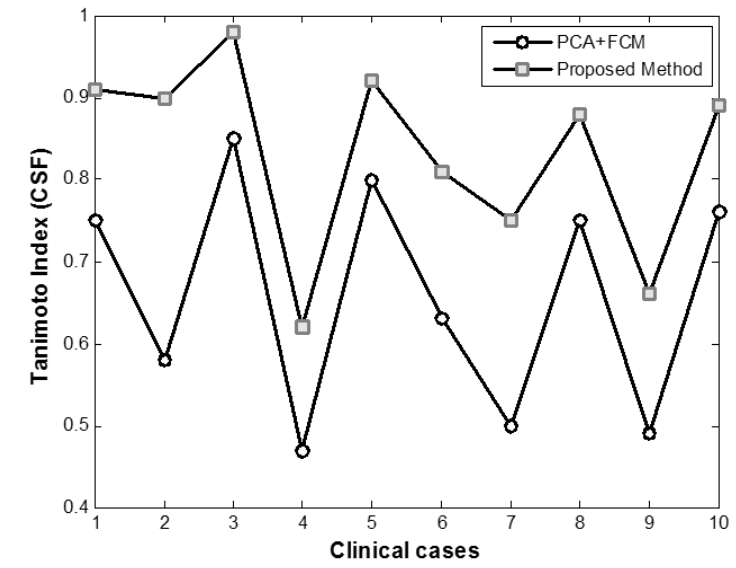



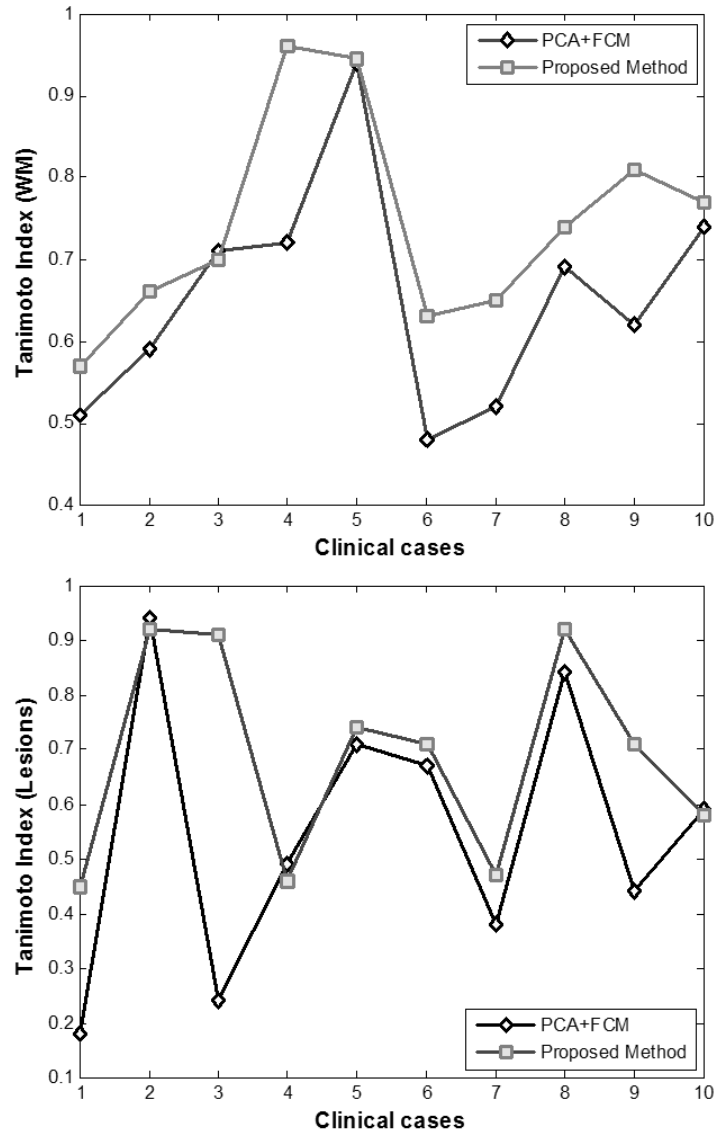

Figure 6. Tanimoto index variations for 10 clinical cases for CSF (top row), WM (middle row) and Lesions (last row)

CSF, TI values from proposed method dominate PCA based classification results in all cases. On an average, new method could keep the value above 0.8 for CSF. However, in the case of WM, PCA based method sometimes keeps the same level with proposed method. Variation in small abnormalities is very noticeable and promising in this work. From the last row, it can be seen that a wide gap exists between two methods for case no. 3 , TI value from proposed method shows a positive difference $>0.65$.

Experiments on synthetic and clinical data gave solid evidence for efficiency of wavelet based PCA in brain tissue classification with improved results, especially in the case of small abnormalities. Novelty of the algorithm lies in using high frequency characteristics to keep local features, and appending detail coefficients with input multisignals to improve the analysis. Classified results using FCM demonstrated the efficiency of the new method by effectively locating small abnormalities, and their effect on other brain tissues. However, the proposed method has to be fine-tuned with optimal parameter settings, and its performance in supervised brain tissue analysis is to be evaluated in future studies.

\section{CONCLUSION}

A multisignal wavelet based PCA is proposed in this work to improve the brain tissue analysis from multispectral MRI. The algorithm is innovative in the way it handles small abnormalities in MRI images. The positive impact of the new method in brain tissue analysis is evaluated by FCM clustering. Experiments on synthetic and clinical data supported the proposed method with improved visual and quantitative results for all reproduced brain tissues. The new method is a promising approach in brain tissue classification for clinical trials. In future, proposed wavelet based PCA can be combined with efficient supervised classification methods like SVM to increase clinical accuracy of multispectral MRI analysis.

\section{ACKNOWLEDGMENT}

Our sincere thanks to Institute of Radiology and Imaging Sciences (IRIS) Pvt. Ltd, Kochi for supporting us with required medical guidance in this work.

\section{REFERENCES}

[1] Vannier M W, Butterfield R L, Jordan D, Murphy W A, Levitt R G, Gado M, Multispectral analysis of magnetic resonance images, Radiology, 1985, 154(1):221-224.

[2] Kvinnsland Y, Brekke N, Taxt T M, Gruner R, Multispectral analysis of multimodal images, Acta Oncol, 2009, 48( 2): 277-84.

[3] Christopher M. Bishop, Pattern Recognition and Machine Learning (Information Science and Statistics), Springer, 2006.

[4] Aapo Hyvarinen, Juha Karhunen, Erkki Oja, Independent component analysis, John Wiley \& Sons Inc, New York, 2001.

[5] Daniel D. Lee, H. Seung, Learning the parts of objects by non-negative matrix factorization, Nature, 1999, 401:788-791.

[6] Stephan Liwicki, Georgios Tzimiropoulos, Stefanos Zafeiriou, Maja Pantic, Euler Principal Component Analysis, Int J Comput Vis, 2013, 101(3):498-518.

[7] Tomoko Tateyama, Zensho Nakao, Yen-Wei Chen, Classification of Brain Matters in MRI by Kernel Independent Component Analysis, Proc of IIH-MSP, IEEE Computer Society, 2008:713-716.

[8] Henry Han, Xiao-Li Li, Multi-resolution independent component analysis for highperformance tumor classification and biomarker discovery, BMC Bioinformatics, 2011, 12(1:S7).

[9] Abdullah N, Lee Wee Chuen, Ngah U K, Ahmad K A, Improvement of MRI brain classification using principal component analysis, Proc IEEE Int Conf on Control System, Computing and Engineering (ICCSCE), 2011:557-561.

[10] Eyal E, Bloch B N, Rofsky N M, Furman-Haran E, Genega E M, Lenkinski R E, Degani H., Principal Component Analysis for Dynamic ContrastEnhanced MRI in Human Prostate Cancer, Invest Radiol, 2010, 45(4):174-181.

[11] Mark-John Bruwer, John F. MacGregor, Michael D. Noseworthy, Dynamic Contrast-Enhanced MRI 
Diagnostics in Oncology via PCA, Proc 10th Scandinavian Symposium on Chemometrics, SSC10, J. Chemometrics, 2008, (22):708-716.

[12] Tiranee Achalakul, Stephen Taylor, A Distributed Spectral-screening PCT Algorithm, J Parallel Distrib Comput, 2003, 63(3):373-384.

[13] Sindhumol S., M. Wilscy, Hyper-Spectral Image Analysis-A Robust Algorithm using Support Vectors and Principal Components, Proc IEEE Int Conf on Computing: Theory and Applications, 2007:389-395,.

[14] Sinthop Kaewpijit, Jacqueline Le Moigne, Tarek ElGhazawi, Feature reduction of hyperspectral imagery using hybrid wavelet principal component analysis, Opt Eng, 2004, 43(2):350-362.

[15] Bruce L M, Koger C H, Jiang Li, Dimensionality reduction of hyperspectral data using discrete wavelet transform feature extraction, IEEE Trans on Geoscience and Remote Sensing, 2002, 40(10):2331-2338.

[16] He R, Datta S, Sajja B R, Narayana P A, Generalized Fuzzy Clustering for Segmentation of Multi-Spectral Magnetic Resonance Images, Comput Med Imaging Graph, 2008, 32(5):353-366.

[17] James C. Bezdek, Pattern Recognition with Fuzzy Objective Function Algorithms, Plenum Press, New York, 1981.

[18] Brainweb:Simulated Brain Database, McConnell Brain Imaging Centre, Montreal Neurological Institute, McGill University. http://www.bic.mni.mcgill.ca/brainweb.

[19] S. Mallat, A Wavelet Tour of Signal Processing, 3rd ed., The Sparse Way Academic Press, 2008.

[20] Sindhumol S, Anil Kumar, Kannan Balakrishnan, Abnormality detection from multispectral brain MRI using multiresolution independent component analysis, International Journal of Signal processing, Image processing, and Pattern recognition, 2013, 6(1):177-190.

[21] Sumitra N, Rakesh Kumar Saxena, Brain Tumor Classification Using Back Propagation Neural Network, International Journal of Image, Graphics and Signal Processing, 2013, 5(2):45-50.

[22] Yang X, Fei B, A multiscale and multiblock fuzzy C-means classification method for brain MR images, Medical Physics, 2011, 38(6):2879-2891.

[23] Boudraa A O, Dehak S M, Zhu Y M, Pachai C, Bao Y G, Grimaud J, Automated segmentation of multiple sclerosis lesions in multispectral MR imaging using fuzzy clustering, Comput Biol Med, 2000, 30(1):23-40.

[24] Jiawei Han, Micheline Kamber, Data Mining: Concepts and Techniques, $2^{\text {nd }}$ Edn., Morgan Kaufmann Publisher, San Fransisco, USA, 2006.

Sindhumol S. received her M.Tech degree in Digital Image Processing from University of Kerala, Trivandrum, India in 2005, and is currently pursuing Ph.D. degree in medical image analysis from Cochin University of
Science and Technology, Kochi, India. She has been working with audio/video/image processing algorithms in IT industry for last 8 years. Her research interest includes multimedia and streaming, multispectral analysis, wavelets, medical imaging, pattern recognition and classification.

Dr. Anil Kumar is a Senior Consultant Radiologist at IRIS, Medall Centre in Kochi, India. He is also working as Associate Professor in Department of Radio diagnosis and Nuclear medicine at Amrita institute of medical sciences, Kochi. He received his MBBS from Medical College, Trivandrum, MD (Radio Diagnosis) from Army Hospital Research and DNB from National Board of Examination, New Delhi. He has over 17 years of experience in Radiology. He has several publications in various national and international journals.

Dr. Kannan Balakrishnan received his M.Sc and M. Phil degrees in Mathematics from University of Kerala, India, M. Tech degree in Computer and Information Science from Cochin University of Science \& Technology (CUSAT), Cochin, India and $\mathrm{Ph} . \mathrm{D}$ in Futures Studies from University of Kerala, India. He is currently working with CUSAT, Cochin, India, as Associate Professor in the Department of Computer Applications. He has published several papers in various national and international journals. His present areas of interest are Graph Algorithms, Intelligent Systems, Image Processing, CBIR and Machine Translation. He is a reviewer of American Mathematical Reviews. 